\title{
Restrained Edge Domination Number of Some Path Related Graphs
}

\author{
S. K. Vaidya ${ }^{1 *}$, P. D. Ajani ${ }^{2}$ \\ ${ }^{1}$ Department of Mathematics, Saurashtra University, Rajkot-360005, India \\ ${ }^{2}$ Department of Mathematics, Atmiya University, Rajkot-360005, India
}

Received 6 August 2020, accepted in final revised form 11 September 2020

\begin{abstract}
For a graph $G=(V, E)$, a set $S \subseteq V(S \subseteq E)$ is a restrained dominating (restrained edge dominating) set if every vertex (edge) not in $S$ is adjacent (incident) to a vertex (edge) in $S$ and to a vertex (edge) in $V-S(E-S)$. The minimum cardinality of a restrained dominating (restrained edge dominating) set of $G$ is called restrained domination (restrained edge domination) number of $G$, denoted by $\gamma_{r}(G)\left(\gamma_{r e}(G)\right)$. The restrained edge domination number of some standard graphs are already investigated while in this paper the restrained edge domination number like degree splitting, switching, square and middle graph obtained from path.
\end{abstract}

Keywords: Dominating set; Restrained dominating set; Restrained edge dominating set; Restrained edge domination number.

(C) 2021 JSR Publications. ISSN: 2070-0237 (Print); 2070-0245 (Online). All rights reserved. doi: http://dx.doi.org/10.3329/jsr.v13i1.48520

J. Sci. Res. 13 (1), 145-151 (2021)

\section{Introduction}

The theory of domination in graphs has received considerable attention due to its diversified applications to handle many real life situations such as design analysis of network to military surveillance and linear algebra to social sciences.

The graphs considered here are simple, finite, connected, undirected with vertex set $V$ and edge set $E$. The minimum degree among the vertices of graph $G$ is denoted by $\delta(G)$ while the maximum degree among the vertices of graph $G$ is denoted by $\Delta(G)$. The open neighborhood of $v \in V$ is the set $N(v)=\{u \in V / u v \in E\}$. An edge $e$ of a graph $G$ is said to be incident with the vertex $v$ if $v$ is an end vertex of $e$.

A set $S \subseteq V$ is a dominating set if every vertex of $V$ is either in $S$ or is adjacent to atleast one element of $S$. The minimum cardinality of a dominating set of $G$ is called domination number and it is denoted by $\gamma(G)$. The edge analogue of dominating set is also available. A subset $F \subseteq E$ is an edge dominating set if each edge in $E$ is either in $F$ or is adjacent to an edge in $F$. An edge dominating set $F$ is called a minimal edge dominating set if no proper subset $F^{\prime}$ of $F$ is an edge dominating set. The edge domination number

\footnotetext{
${ }^{*}$ Corresponding author: samirkvaidya@yahoo.co.in
} 
$\gamma_{e}(G)$ is the minimum cardinality among all minimal edge dominating sets. The concept of edge domination was introduced by Mitchell and Hedetniemi [1]. Vaidya and Pandit [2] have discussed edge domination in some path and cycle related graphs and edge domination in graphs is explored by Arumugam and Velammal [3].

For graph theoretic terminology and notation we rely on Harary [4]. A brief account of dominating set and its related concepts can be found in Haynes et al. [5]. Many variants of dominating sets are available in the existing literature. One such variant is restrained domination, a set $S \subseteq V$ is a restrained dominating set if every vertex not in $S$ is adjacent to a vertex in $S$ as well as to a vertex in $V-S$. The minimum cardinality of a restrained dominating set $S$ is called the restrained domination number of $G$, denoted by $\gamma_{r}(G)$. The concept of restrained domination was introduced by Telle and Proskurowski [6] as a vertex partitioning problem. Restrained domination in the context of path and cycle is discussed by Vaidya and Ajani [7,8] while the restrained domination of complete graph, multipartite graphs and the graphs with minimum degree two is well studied by Domke et al. $[9,10]$. Moreover the concept of total equitable bondage number was introduced by Vaidya and Parmar [11,12] while equi independent equitable domination was explored by Vaidya and Kothari [13]. These variants are introduced by identifying one or more characteristics of elements of vertex subset or edge subset.

The present work is focused on edge analogue of restrained domination in graphs. For a graph $G=(V, E)$, a set $F \subseteq E$ is a restrained edge dominating set if every edge not in $F$ is incident to an edge in $F$ and also incident to an edge in $E-F$. The minimum cardinality of restrained edge dominating set of $G$ is called restrained edge domination number, denoted as $\gamma_{r e}(G)$. This concept was introduced by Soner and Ghobadi [14] and further explored by Vaidya and Ajani [15].

The restrained edge domination numbers are investigated for the graphs obtained from degree splitting, switching of a vertex, square and middle graph of path.

Definition 1.1: The degree of an edge $e=u v$ of $G$ is defined by $\operatorname{deg}(e)=\operatorname{deg}(u)+$ $\operatorname{deg}(v)-2$ and it is equal to the number of edges adjacent to it. The maximum degree of an edge in $G$ is denoted by $\Delta^{\prime}(G)$.

Definition 1.2: Let $G=(V, E)$ be a graph with $V(G)=S_{1} \cup S_{2} \cup S_{3} \cup \ldots \cup S_{t} \cup T$, where each $S_{i}$ is a set of all the vertices of same degree with at least two elements and $T=V(G)-\bigcup_{i=1}^{t} S_{i}$. The degree splitting graph $D S(G)$ is obtained from $\mathrm{G}$ by adding vertices $w_{1}, w_{2}, w_{3}, \ldots, w_{t}$ and joining $w_{i}$ to each vertex of $S_{i}$ for $1 \leq i \leq t$. Note that if $V(G)=\cup_{i=1}^{t} S_{i}$ then $T=\phi$.

Definition 1.3: The switching of a vertex $v$ of $G$ means removing all the edges incident to $v$ and adding edges joining $v$ to every vertex which is not adjacent to $v$ in $G$. We denote the resultant graph by $\tilde{G}$.

Definition 1.4: The square of a graph $G$ denoted by $G^{2}$ has the same vertex set as of $G$ and two vertices are adjacent in $G^{2}$ if they are at distance of 1 or 2 apart in $G$. 


\section{Main Results}

Theorem 2.1: If $\left(D S\left(P_{n}\right)\right)$ is the graph obtained by degree splitting of $P_{n}$ then,

$$
\gamma_{r e}\left(D S\left(P_{n}\right)\right)=\left\lceil\frac{n+2}{3}\right\rceil \text {. }
$$

Proof: Let $v_{1}, v_{2}, \ldots, v_{n}$ be the vertices and $e_{1}, e_{2}, \ldots, e_{n-1}$ be the edges of $P_{n}$. Let $S_{1}$ and $S_{2}$ be the set of all the vertices of same degree with at least two elements. In order to obtain $D S\left(P_{n}\right)$ from path $P_{n}$. We add two vertices $u$ and $v$ corresponding to $S_{1}$ and $S_{2}$ such that each vertex of $S_{1}$ and $S_{2}$ joined by an edge to $u$ and $v$ respectively. Let $e_{1}^{\prime}, e_{2}^{\prime}, \ldots, e_{n}^{\prime} \in$ $E\left(D S\left(P_{n}\right)\right)$, where $e_{1}^{\prime}=u v_{1}, e_{n}^{\prime}=u v_{n}$ and $e_{i}^{\prime}=v v_{i}$ for $i=2,3, \ldots,(n-1) \cdot\left|E\left(D S\left(P_{n}\right)\right)\right|=$ $2 n-1$.

Now the edge sets $F=\left\{e_{1}^{\prime}, e_{2}^{\prime}\right\}$ and $F=\left\{e_{1}, e_{3}\right\}$ are clearly restrained edge dominating sets with minimum cardinality of $D S\left(P_{3}\right)$ and $D S\left(P_{4}\right)$ respectively. Hence $\gamma_{\text {re }}=2=\left\lceil\frac{n+2}{3}\right\rceil$, for $n=3,4$.

For $n \geq 5$, we construct an edge set of $D S\left(P_{n}\right)$ as follows.

$F=\left\{\begin{array}{ccc}\left\{e_{1}, e_{4}, \ldots, e_{3 i+1}\right\} \cup\left\{e_{n-1}\right\} \cup\left\{e_{n-3}^{\prime}\right\}, & \text { where } 0 \leq i \leq\left\lceil\frac{n-4}{3}\right\rceil, & \text { for } n \equiv 0 \text { or } 1(\bmod 3) \\ \left\{e_{1}, e_{4}, \ldots, e_{3 i+1}\right\} \cup\left\{e_{n-3}^{\prime}\right\}, & \text { where } 0 \leq i \leq\left\lfloor\frac{n-1}{3}\right], & \text { for } n \equiv 2(\bmod 3)\end{array}\right.$ with $|F|=\left\lceil\frac{n+2}{3}\right\rceil$. Since $F \subseteq E\left(D S\left(P_{n}\right)\right)$ and each edge in $E-F$ is adjacent to an edge in $F$ and another edge in $E-F$, the above set $F$ is a restrained edge dominating set of $D S\left(P_{n}\right)$. Moreover the above set $F$ is a minimal restrained edge dominating set of $D S\left(P_{n}\right)$ because for any edge $e \in F$, the set $F-\{e\}$ does not dominate the edges in $N(e)$ of $D S\left(P_{n}\right)$.

Now $\operatorname{deg}\left(e_{i}^{\prime}\right)=n-1=\Delta^{\prime}\left(D S\left(P_{n}\right)\right)$, for $2 \leq i \leq n-1$ and $\operatorname{deg}\left(e_{1}\right)=3=\operatorname{deg}\left(e_{n-1}\right)$ also $\operatorname{deg}\left(e_{i}\right)=4$, for $2 \leq i \leq n-2$. So the edges in above set $F$ will dominate maximum number of distinct edges of $D S\left(P_{n}\right)$. Therefore any set containing the edges less than the number of edges in set $F$ will not dominate all the edges of $D S\left(P_{n}\right)$. This implies that above set $F$ is the restrained edge dominating set with minimum cardinality.

Hence set $F$ is a minimal restrained edge dominating set with minimum cardinality among all the minimal restrained edge dominating set of $D S\left(P_{n}\right)$. Thus, $\gamma_{r e}\left(D S\left(P_{n}\right)\right)=$ $\left\lceil\frac{n+2}{3}\right\rceil$.

Illustration 2.2: The degree splitting of $P_{7}$ is shown in Fig. 1 where the set of edges $\left\{e_{1}, e_{6}, e_{4}^{\prime}\right\}$ is its restrained edge dominating set of minimum cardinality. 


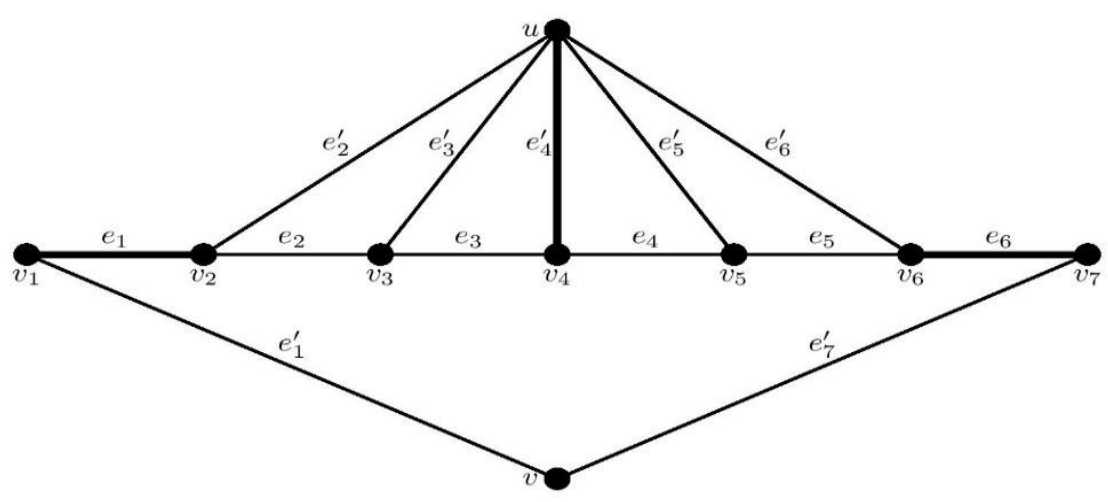

Fig. 1. $\gamma_{r e}\left(D S\left(P_{7}\right)\right)=3$

Theorem 2.3: If $\widetilde{P_{n}}$ is the graph obtained by switching of a pendant vertex $P_{n}$ then,

$\gamma_{r e}\left(\widetilde{P_{n}}\right)=\left\{\begin{array}{cl}2 ; & \text { for } n=3 \\ \left\lceil\frac{n-1}{3}\right\rceil ; & \text { for } n \geq 4\end{array}\right.$

Proof: Let $P_{n}$ be the path of order $n$ with vertex set $V\left(P_{n}\right)=\left\{v_{1}, v_{2}, v_{3}, \ldots, v_{n}\right\}$ and edge set $E\left(P_{n}\right)=\left\{e_{1}, e_{2}, e_{3}, \ldots, e_{n-1}\right\}$. Let $\widetilde{P_{n}}$ be the graph obtained by switching of a pendant vertex $v_{1}$ of $P_{n}$. Then $\left|V\left(\widetilde{P_{n}}\right)\right|=n$ and $\left\{e_{1}, e_{2}, e_{3}, \ldots, e_{n-1}, e_{1}^{\prime}, e_{2}^{\prime}, \ldots, e_{n-2}^{\prime}\right\} \in E\left(\widetilde{P_{n}}\right)$ with $\left|E\left(\widetilde{P_{n}}\right)\right|=2 n-4$.

Now the edge set $F=\left\{e_{1}^{\prime}, e_{2}\right\}$ is clearly a restrained edge dominating set of $\widetilde{P_{3}}$ with minimum cardinality. Hence $\gamma_{r e}\left(\widetilde{P_{3}}\right)=2$, for $n=3$.

For $n \geq 4$, we construct an edge set of $\widetilde{P_{n}}$ as follows,

$F=\left\{\begin{array}{ccc}\left\{e_{5}, e_{8}, \ldots, e_{3 i+2}\right\} \cup\left\{e_{1}^{\prime}\right\}, & \text { where } 1 \leq i \leq\left\lceil\frac{n-4}{3}\right\rceil, & \text { for } n \equiv 0 \text { or } 1(\bmod 3) \\ \left\{e_{5}, e_{8}, \ldots, e_{3 i+2}\right\} \cup\left\{e_{n-1}\right\} \cup\left\{e_{1}^{\prime}\right\}, & \text { where } 1 \leq i \leq\left\lceil\frac{n-4}{3}\right], & \text { for } n \equiv 2(\bmod 3)\end{array}\right.$

with $|F|=\left\lceil\frac{n-1}{3}\right\rceil$. Note that $F \subseteq E\left(\widetilde{P_{n}}\right)$. As each edge in $E-F$ is adjacent to an edge in $F$ and to another edge in $E-F$, it follows that $F$ is a restrained edge dominating set of $\widetilde{P_{n}}$. For any edge $e \in F$, the set $F-\{e\}$ does not dominate the edges in $N(e)$ of $\widetilde{P_{n}}$, which implies that the above set $F$ is a minimal restrained edge dominating set of $\widetilde{P_{n}}$.

Now $\operatorname{deg}\left(e_{i}^{\prime}\right)=n-1=\Delta^{\prime}\left(\widetilde{P}_{n}\right)$, for $2 \leq i \leq n-1$ and $\operatorname{deg}\left(e_{n-1}\right)=3$, also $\operatorname{deg}\left(e_{i}\right)=4$, for $2 \leq i \leq n-1$. Due to adjacency nature, the edges in above set $F$ will dominate maximum number of distinct edges of $\widetilde{P}_{n}$. Therefore any set containing the edges less than the number of edges in the set $F$ will not dominate all the edges of $\widetilde{P_{n}}$. This implies that above set $F$ is the restrained edge dominating set with minimum cardinality.

Hence set $F$ is a minimal restrained edge dominating set with minimum cardinality among all the minimal restrained edge dominating set of $\widetilde{P_{n}}$. Thus $\gamma_{r e}\left(\widetilde{P_{n}}\right)=\left\lceil\frac{n-1}{3}\right\rceil$, for $n \geq 4$. 
Illustration 2.4: The switching of path $P_{6}$ is shown in Fig. 2 where the set of edges $\left\{e_{1}^{\prime}, e_{5}\right\}$ is its restrained edge dominating set of minimum cardinality.

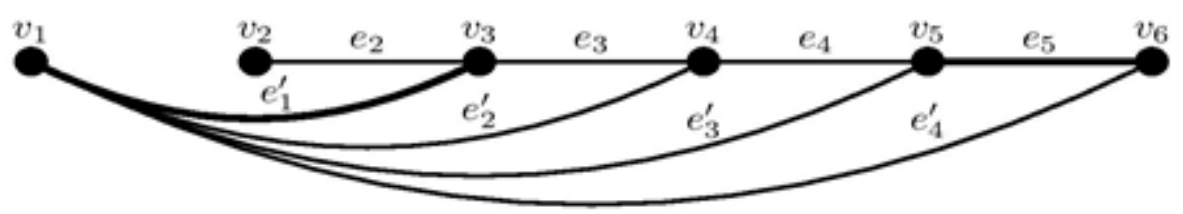

Fig. 2. $\gamma_{r e}\left(\widetilde{P_{6}}\right)=2$.

Theorem 2.5: If $P_{n}^{2}$ is the graph obtained from square of path $P_{n}$ then $\gamma_{r e}\left(P_{n}^{2}\right)=\left\lceil\frac{n-1}{3}\right\rceil$.

Proof: Let $P_{n}$ be a path of order $n$ with $E\left(P_{n}\right)=\left\{e_{1}, e_{2}, \ldots, e_{n-1}\right\}$. Let $P_{n}^{2}$ be the graph obtained from $P_{n}$ such that $\left|V\left(P_{n}\right)\right|=\left|V\left(P_{n}^{2}\right)\right|=n$ and $\left|E\left(P_{n}^{2}\right)\right|=2 n-3$ with edge set $\left\{e_{1}, e_{2}, \ldots, e_{n-1}, e_{1}^{\prime}, e_{2}^{\prime}, \ldots, e_{n-2}^{\prime}\right\} \in E\left(P_{n}^{2}\right)$.

Now the edge set $F=\left\{e_{1}\right\}$ or $\left\{e_{2}\right\}$ and $F=\left\{e_{2}\right\}$ are clearly restrained edge dominating set of $P_{3}^{2}$ and $P_{4}^{2}$ with minimum cardinality respectively. Hence $\gamma_{r e}\left(P_{n}^{2}\right)=$ $1=\left\lceil\frac{n-1}{3}\right\rceil$, for $n=3,4$.

For $n \geq 5$, we construct an edge set of $P_{n}^{2}$ as follows,

$F=\left\{\begin{array}{ccc}\left\{e_{2}, e_{5}, e_{8}, \ldots, e_{3 i+2}\right\}, & \text { where } 0 \leq i \leq\left\lceil\frac{n-4}{3}\right\rceil, & \text { for } n \equiv 0 \text { or } 1(\bmod 3) \\ \left\{e_{2}, e_{5}, e_{8}, \ldots, e_{3 i+2}\right\} \cup\left\{e_{n-1}\right\}, & \text { where } 0 \leq i \leq\left\lceil\frac{n-4}{3}\right], & \text { for } n \equiv 2(\bmod 3)\end{array}\right.$

with $|F|=\left\lceil\frac{n-1}{3}\right\rceil$. Since $F \subseteq E\left(P_{n}^{2}\right)$ and each edge in $E-F$ is adjacent to an edge in $F$ and to another edge in $E-F$. It follows that the set $F$ is a restrained edge dominating set of $P_{n}^{2}$. Moreover the above set $F$ is a minimal restrained edge dominating set of $P_{n}^{2}$ because for any edge $e \in F$, the set $F-\{e\}$ does not dominate the edges in $N(e)$ of $P_{n}^{2}$.

Now $\operatorname{deg}\left(e_{i}\right)=6=\Delta^{\prime}\left(P_{n}^{2}\right)$, for $3 \leq i \leq n-3$ and $\operatorname{deg}\left(e_{2}\right)=5=\operatorname{deg}\left(e_{n-2}\right)$. So the edges in the set $F$ will dominate maximum number of distinct edges of $P_{n}^{2}$. Therefore any set containing the edges less than the number of edges in the set $F$ will not dominate all the edges of $P_{n}^{2}$. This implies that above set $F$ is the restrained edge dominating set with minimum cardinality.

Hence set $F$ is a minimal restrained edge dominating set with minimum cardinality among all the minimal restrained edge dominating set of $P_{n}^{2}$. Thus, $\gamma_{r e}\left(P_{n}^{2}\right)=\left\lceil\frac{n-1}{3}\right\rceil$

Illustration 2.6: The square of path $P_{7}$ is shown in Fig. 3 where the set of edges $\left\{e_{2}, e_{5}\right\}$ is its restrained edge dominating set of minimum cardinality. 


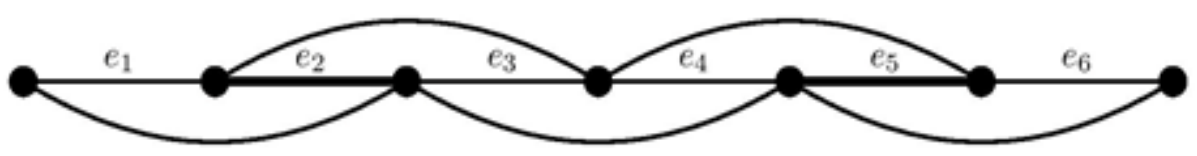

Fig. 3. $\gamma_{r e}\left(P_{7}^{2}\right)=2$

Theorem 2.7: If $M\left(P_{n}\right)$ is the middle graph of $P_{n}$ then $\gamma_{r e}\left(M\left(P_{n}\right)\right)=\left\{\begin{array}{cl}2 ; & \text { for } n=2 \\ \left\lfloor\frac{n}{2}\right\rfloor ; & \text { for } n \geq 3\end{array}\right.$.

Proof: Let $P_{n}$ be a path of order $n$ with $V\left(P_{n}\right)=\left\{u_{1}, u_{2}, u_{3}, \ldots, u_{n}\right\}$. In order to obtain $M\left(P_{n}\right)$, we add vertices $u_{1}^{\prime}, u_{2}^{\prime}, u_{3}^{\prime}, \ldots, u_{n-1}^{\prime}$ corresponding to the edges $f_{1}, f_{2}, f_{3}, \ldots, f_{n-1}$ of $P_{n}$. Thus, $V\left(M\left(P_{n}\right)\right)=\left\{u_{1}, u_{2}, u_{3}, \ldots, u_{n}, u_{1}^{\prime}, u_{2}^{\prime}, u_{3}^{\prime}, \ldots, u_{n-1}^{\prime}\right\}$ and $E\left(M\left(P_{n}\right)\right)=\left\{e_{1}, e_{2}, e_{3} \ldots, e_{2 n-2}, e_{1}^{\prime}, e_{2}^{\prime}\right.$,

$e_{3}^{\prime} \ldots, e_{n-2}^{\prime}$, where $e_{j}^{\prime}=u_{j}^{\prime} u_{j+1}^{\prime}$ for $j=1,2, \ldots, n-2$. Note that $\left|V\left(M\left(P_{n}\right)\right)\right|=2 n-1$ and $\left|E\left(M\left(P_{n}\right)\right)\right|=3 n-4$.

Now the edge set $F=\left\{e_{1}, e_{2}\right\}$ is clearly a restrained edge dominating set of $M\left(P_{2}\right)$ with minimum cardinality. So $\gamma_{r e}\left(M\left(P_{2}\right)\right)=2$, for $n=2$.

For $n \geq 3$, we construct an edge set of $M\left(P_{n}\right)$ as follows, $F=\left\{e_{1}^{\prime}, e_{3}^{\prime}, e_{5}^{\prime}, \ldots, e_{2 i+1}^{\prime}\right\} \cup\left\{e_{n-2}^{\prime}\right\}$, where $0 \leq i \leq\left\lfloor\frac{n-4}{2}\right\rfloor$ with $|F|=\left\lfloor\frac{n}{2}\right\rfloor$. Note that $F \subseteq$ $M\left(P_{n}\right)$. As each edge in $E-F$ is adjacent to an edge in $F$ and to another edge in $E-F$, it follows that $F$ is a restrained edge dominating set of $M\left(P_{n}\right)$. Moreover $\operatorname{deg}\left(e_{i}^{\prime}\right)=6=$ $\Delta^{\prime}\left(M\left(P_{n}\right)\right)$, for $2 \leq i \leq n-3$ and $\operatorname{deg}\left(e_{i}\right)=4$, for $3 \leq i \leq 2 n-4$.

We claim that above set $F$ is a restrained edge dominating set with minimum cardinality. If possible, suppose $F^{\prime}$ is a restrained edge dominating set of $M\left(P_{n}\right)$ such that $\left|F^{\prime}\right|=\left\lfloor\frac{n}{2}\right\rfloor-1<\left\lfloor\frac{n}{2}\right\rfloor=|F|$. In order to attain minimum cardinality, $F^{\prime}$ does not contain the edges where each edge among them can dominate distinct $3 n-4$ edges of $M\left(P_{n}\right)$. Moreover $\Delta^{\prime}\left(M\left(P_{n}\right)\right) \cdot\left\lfloor\frac{n}{2}\right\rfloor-1=6 .\left(\left\lfloor\frac{n}{2}\right\rfloor-1\right)<3 n-4=\left|E\left(M\left(P_{n}\right)\right)\right|$. Therefore $F^{\prime}$ can not be a restrained edge dominating set of $M\left(P_{n}\right)$. Which implies that $F$ is a restrained edge dominating set of $M\left(P_{n}\right)$ with minimum cardinality. Hence, $\gamma_{r e}\left(M\left(P_{n}\right)\right)=\left\lfloor\frac{n}{2}\right\rfloor$, for $n \geq 3$.

Illustration 2.8: The middle graph of path $P_{7}$ is shown in Fig. 4 where the set of edges $\left\{e_{1}^{\prime}, e_{3}^{\prime}, e_{5}^{\prime}\right\}$ is its restrained edge dominating set of minimum cardinality.

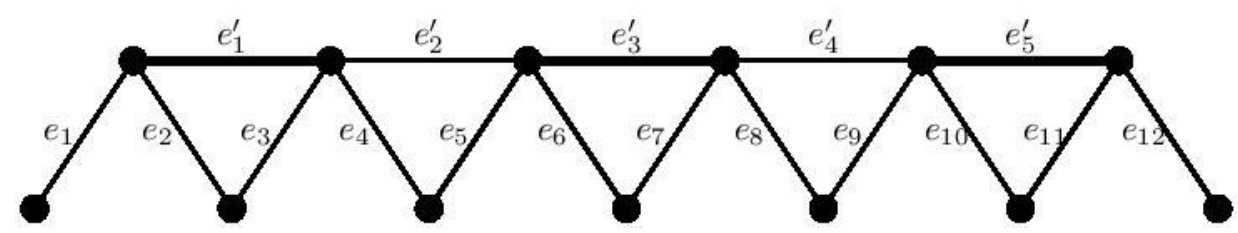

Fig. 4. $\gamma_{r e}\left(M\left(P_{7}\right)\right)=3$. 


\section{Conclusion}

There are many variants of edge dominating sets available in the literature and study of related dominating parameters is also interesting. The restrained edge dominating set relates the edge set and its complements. This dominating parameter have been studied for degree splitting, switching, square and middle graph of path. To investigate such parameters for various graphs obtained from standard graphs is an open area of research.

\section{References}

1. S. Mitchell and S. Hedetniemi, Congr. Numer. 19, 489 (1977).

2. S. K. Vaidya and R. M. Pandit, ISRN Discrete Math. 2014, 1 (2014). https://doi.org/10.1155/2014/486354

3. S. Arumugam and S. Velammal, Taiwanese J. Math. 2, 173 (1998). https://doi.org/10.11650/twjm/1500406930

4. F. Harary, Graph Theory (Addison-Wesley, Massachusetts, 1969). https://doi.org/10.21236/AD0705364

5. T. W. Haynes, S. T. Hedetniemi, and P. J. Slater, Fundamentals of Domination in Graphs (Marcel Dekker, New York, 1998). https://doi.org/10.1201/9781482246582

6. J. A. Telle and A. Proskurowski, SIAM J. Discrete Math. 10, 529 (1997). https://doi.org/10.1137/S0895480194275825

7. S. K. Vaidya and P. D. Ajani, J. Comput. Math. 1, 114 (2017). https://doi.org/10.26524/cm10

8. S. K. Vaidya and P. D. Ajani, Int. J. Math. Soft Comput. 8, 17 (2018). https://doi.org/10.26708/IJMSC.2017.1.8.03

9. G. S. Domke, J. H. Hattingh, S. T. Hedetiniemi, R. C. Laskar, and L. R. Markus, Discrete Math. 203, 1 (1999). https://doi.org/10.1016/S0012-365X(99)00016-3

10. G. S. Domke, J. H. Hattingh, M. A. Henning, and L. R. Markus, J. Combin. Math. Combin. Comput. 35, 239 (2000).

11. S. K. Vaidya and A. D. Parmar, J. Sci. Res. 10, 231 (2018). https://doi.org/10.3329/jsr.v10i3.33940

12. S. K. Vaidya and A. D. Parmar, J. Sci. Res. 11, 303 (2019). https://doi.org/10.3329/jsr.v11i3.40573

13. S. K. Vaidya and N. J. Kothari, J. Sci. Res. 7, 77 (2015). https://doi.org/10.3329/jsr.v7i3.23412

14. N. D. Soner and S. Ghobadi, Adv. Stud. Contemp. Math. 19, 143 (2009).

15. S. K. Vaidya and P. D. Ajani, Malaya J. Matematik 8, 28 (2020). https://doi.org/10.26637/MJM0801/0005 